\title{
Licochalcone A induces apoptosis in KB human oral cancer cells via a caspase-dependent FasL signaling pathway
}

\author{
JAE-SUNG KIM ${ }^{1}$, MI-RA PARK ${ }^{1}$, SOOK-YOUNG LEE ${ }^{1}$, DO KYOUNG KIM ${ }^{1,2}$, SUNG-MIN MOON ${ }^{3}$, \\ CHUN SUNG KIM ${ }^{3}$, SEUNG SIK CHO ${ }^{5}$, GOO YOON ${ }^{5}$, HEE-JEONG IM ${ }^{6}$, \\ JAE-SEEK YOU ${ }^{4}$, JI-SU OH ${ }^{1,4}$ and SU-GWAN KIM ${ }^{1,4}$ \\ ${ }^{1}$ Regional Innovation Center for Dental Science and Engineering; Departments of ${ }^{2}$ Oral Physiology, ${ }^{3}$ Oral Biochemistry \\ and ${ }^{4}$ Oral and Maxillofacial Surgery, Chosun University, Gwangju 501-759; ${ }^{5}$ Department of Pharmacy, \\ College of Pharmacy, Mokpo National University, Muan, Jeonnam 535-729, Republic of Korea; \\ ${ }^{6}$ Department of Biochemistry, Rush University Medical Center, Chicago, IL 60612, USA
}

Received October 7, 2013; Accepted November 25, 2013

DOI: $10.3892 /$ or.2013.2929

\begin{abstract}
Licochalcone A (Lico-A) is a natural phenol licorice compound with multiple bioactivities, including anti-inflammatory, anti-microbial, anti-fungal and osteogenesis-inducing properties. In the present study, we investigated the Lico-A-induced apoptotic effects and examined the associated apoptosis pathway in KB human oral cancer cells. Lico-A decreased the number of viable KB oral cancer cells. However, Lico-A did not have an effect on primary normal human oral keratinocytes. In addition, the $\mathrm{IC}_{50}$ value of Lico-A was determined to be $\sim 50 \mu \mathrm{M}$ following dose-dependent stimulation. $\mathrm{KB}$ oral cancer cells stimulated with Lico-A for $24 \mathrm{~h}$ showed chromatin condensation by DAPI staining, genomic DNA fragmentation by agarose gel electrophoresis and a gradually increased apoptotic cell population by FACS analysis. These data suggest that Lico-A induces apoptosis in $\mathrm{KB}$ oral cancer cells. Additionally, Lico-A-induced apoptosis in KB oral cancer cells was mediated by the expression of factor associated suicide ligand (FasL) and activated caspase- 8 and -3 and poly(ADP-ribose) polymerase (PARP). Furthermore, in the $\mathrm{KB}$ oral cancer cells co-stimulation with a caspase inhibitor (Z-VAD-fmk) and Lico-A significantly abolished the apoptotic phenomena. Our findings demonstrated that Lico-A-induced apoptosis in KB oral cancer cells involves the extrinsic apoptotic signaling pathway, which involves a caspase-dependent FasL-mediated death receptor pathway. Our data suggest that Lico-A be developed as a chemotherapeutic agent for the management of oral cancer.
\end{abstract}

Correspondence to: Professor Su-Gwan Kim, Department of Oral and Maxillofacial Surgery, School of Dentistry, Chosun University, 375 Seosuk-dong, Dong-gu, Gwangju 501-759, Republic of Korea E-mail: sgckim@chosun.ac.kr

Key words: licochalcone A, oral cancer, apoptosis, Fas ligand, caspase

\section{Introduction}

Licochalcone A [Lico-A; (E)-3-[4-hydroxy-2-methoxy-5-(2methylbut-3-en-2-yl)phenyl]-1-(4-hydroxyphenyl)prop-2-en-1one] is a natural phenol licorice compound isolated from licorice root (Radix Glycyrrhizae). Lico-A has been used for thousands of years as a traditional herbal medicine and is known for its multiple bioactivities including antiinflammatory (1), anti-microbial (2), anti-malarial (3), and osteogenic activity (4), and anti-angiogenic (5) and anticancer effects (6-9). Moreover, Lico-A has also been suggested to be beneficial for common oro-dental disease via its anti-adhesion properties (10). Although the multiple bioactivities of Lico-A have been revealed in various research fields, its anticancer property in oral cancer is still largely unknown.

Oral cancer is a major worldwide public health issue and may affect any region of the oral cavity, including the lips, tongue, mouth and throat $(11,12)$. Although the pathophysiological studies associated with the development of oral cancer have shown that environmental factors, such as smoking, alcohol and betel quid, may act as critical carcinogens, the etiology of oral cancer is still largely unknown (13-15). However, oral cancer is one of the most prevalent cancers with an incidence rate of 3.9 cases per 100,000 individuals worldwide (16). Even though the clinical treatment for oral cancer has shown significant improvement during the past decade, current clinical treatments consisting of surgery and/ or radiotherapy are not totally free from various side-effects, including loss of function and disfigurement. As a result, these side-effects reduce the quality of life of patients with oral cancer. Therefore, various studies are underway to develop effective clinical treatment with minimal side-effects for oral cancer. As part of these studies, intensive research is being carried out to develop novel chemotherapeutic agents from edible herbal plants or natural products (17-21).

Apoptosis is generally defined as programmed cell death via a precisely regulated cell suicide process, which is mediated by different intracellular and extracellular biological mechanisms (22). Apoptosis largely occurs through two pathways; one is the mitochondrial apoptotic pathway and 
the second is the death receptor pathway. The mitochondrial pathway, known as intrinsic apoptotic signaling, is triggered by the release of cytochrome $c$ due to the loss of mitochondrial transmembrane potential $(23,24)$. The death receptor pathway, known as extrinsic apoptotic signaling, is mediated by sequential activation of caspase- 8 and -3 and poly(ADP-ribose) polymerase (PARP), after interaction with death receptor and its ligands, such as TRAIL and factor associated suicide ligand (FasL) (25). Importantly, apoptosis has emerged as an important mechanism for the anticancer effects of chemotherapeutic agents developed from herbal plants.

Hence, the aim of the present study was to determine whether Lico-A has potential to function as a chemotherapeutic agent for the treatment of $\mathrm{KB}$ oral cancer cells without affecting normal cells originating from the oral cavity. Furthermore, the present study aimed to evaluate the potential apoptotic effect of Lico-A and to elucidate the Lico-A-induced apoptotic signaling pathway in KB oral cancer cells.

\section{Materials and methods}

Cell culture. Normal human oral keratinocytes (NHOKs) were purchased from ScienCell Research Laboratories (Carlsbad, CA, USA). The NHOKs were maintained in DMEM (Gibco, Grand Island, NY, USA) containing $10 \%$ fetal bovine serum (FBS) (Invitrogen, Carlsbad, CA, USA) at $37^{\circ} \mathrm{C}$ in an atmosphere containing $5 \% \mathrm{CO}_{2}$. The human oral squamous cell carcinoma cell line, KB, was obtained from the American Type Culture Collection (ATCC; Manassas, VA, USA) and cultured according to the cell culture instructions provided. Briefly, KB cells were grown in MEM containing $10 \% \mathrm{FBS}$ at $37^{\circ} \mathrm{C}$ in an atmosphere containing $5 \% \mathrm{CO}_{2}$.

Cell viability assay. Both $\mathrm{KB}$ oral cancer cells and NHOKs were seeded at a density of $5 \times 10^{5}$ cells/well in 96-well plates and allowed to attach to the well overnight. After incubation, cultured cells were stimulated with various concentrations of Lico-A in triplicate and incubated at $37^{\circ} \mathrm{C}$ in a $5 \%$ humidified $\mathrm{CO}_{2}$ incubator for $24 \mathrm{~h}$. Subsequently, 3-(4,5-dimethylthiazol2-yl)-2,5-diphenyltetrazolium bromide (MTT) was added to each well, and incubation was continued for a further $4 \mathrm{~h}$ at $37^{\circ} \mathrm{C}$. To dissolve the formazan formed from MTT, the cells were resuspended in $200 \mu \mathrm{l}$ dimethyl sulfoxide (DMSO), and the optical density (OD) of the solution was determined using a spectrometer at a wavelength of $570 \mathrm{~nm}$. The experiments were repeated 3 times, independently. The mean optical density $(\mathrm{OD}) \pm \mathrm{SD}$ for each group of replicates was calculated. The entire procedure was repeated 3 times. The inhibitory rate of cell growth was calculated using the equation: \% Growth inhibition $=\left(1-\mathrm{OD}_{\text {extract treated }}\right) / \mathrm{OD}_{\text {negative control }} \times 100$.

Live/dead cell viability assay. The live/dead cell viability assay was carried out as previously described (26), using calcein AM to stain the live cells and ethidium bromide homodimer 1 to stain the dead cells. These reagents were obtained from Molecular Probes (Eugene, OR, USA). For the cell survival assay, KB oral cancer cells and HNOKs were plated in a chamber slide, stimulated with berberine for $24 \mathrm{~h}$ and stained with green calcein AM and ethidium bromide homodimer 1 according to the manufacturer's protocol. The cells were then observed and photographed by inverted phasecontrast microscopy.

DNA fragmentation assay. KB oral cancer cells were collected after stimulation with Lico-A $(0,25$ and $50 \mu \mathrm{M})$ for $24 \mathrm{~h}$ and were rinsed 3 times in phosphate-buffered saline (PBS) at $4^{\circ} \mathrm{C}$. This was followed by degradation using $100 \mu \mathrm{l}$ cell lysate buffer (1\% NP-40, 20 mM EDTA, 50 mM Tris-HCl, pH 7.5) at $4{ }^{\circ} \mathrm{C}$ for $10 \mathrm{~min}$, followed by centrifugation at $12,000 \mathrm{x} \mathrm{g}$ for $30 \mathrm{~min}$. RNase A was added to the supernatant and incubated at $37^{\circ} \mathrm{C}$ for $1 \mathrm{~h}$. Proteinase $\mathrm{K}$ was then added to the supernatant, and incubation was conducted at $37^{\circ} \mathrm{C}$ for $8 \mathrm{~h}$. An equal volume of isopropanol was then added and kept at $-80^{\circ} \mathrm{C}$ for $24 \mathrm{~h}$ to precipitate the genomic DNA. The supernatant was removed after centrifugation at $12,000 \mathrm{x} \mathrm{g}$ for $15 \mathrm{~min}$ at $4^{\circ} \mathrm{C}$. The supernatant was allowed to dry naturally and was dissolved in TE buffer, followed by electrophoresis on $1.5 \%$ agarose gels. A gel imaging system was used for observation and capturing images.

4',6-Diamidino-2-phenylindole (DAPI) staining. KB oral cancer cells stimulated with 25 and $50 \mu \mathrm{M}$ Lico-A for $24 \mathrm{~h}$ were fixed with $4 \%$ paraformaldehyde prior to washing with PBS. The washed cells were then stained with $1 \mathrm{mg} / \mathrm{ml} \mathrm{4',6'-diamidino-}$ 2-phenylindole dihydrochloride (DAPI) (Roche Diagnostics) for $20 \mathrm{~min}$ in the dark. The DAPI-stained images were capturing using fluorescence microscopy (Eclipse TE200; Nikon Instruments, Melville, NY, USA).

Caspase-3/-7 activity assay. The apoptosis executioner caspase-3/-7 activity was determined using the cell-permeable fluorogenic substrate, PhiPhiLux $-\mathrm{G}_{1} \mathrm{D}_{2}$ (Oncoimmunin Inc., Gaithersburg, MD, USA), according to the manufacturer's instructions.

Annexin V-FITC and propidium iodide (PI) staining and flow cytometric analysis. Apoptosis was determined by Annexin V-fluorescein isothiocyanate assay. Cells were washed in PBS twice and resuspended in binding buffer (BD Biosciences, San Diego, CA, USA). Annexin V-fluorescein isothiocyanate and 7-amino-actinomycin $\mathrm{D}$ (BD Biosciences) were added to the cells, which were then incubated in the dark for $15 \mathrm{~min}$. Cells were then added and resuspended in $400 \mathrm{ml}$ of binding buffer. Cells were analyzed using a fluorescence activated cell sorting FACSCalibur flow cytometer (BectonDickinson, San Jose, CA, USA). Data analysis was performed using standard CellQuest software (Becton-Dickinson).

RNA isolation and quantitative PCR analysis. Total RNA was isolated using TRIzol reagent (Life Technologies, Carlsbad, CA, USA), according to the manufacturer's instructions. Total RNA $(1 \mu \mathrm{g})$ was reverse transcribed into first strand cDNA using ThermoScript ${ }^{\mathrm{TM}}$ RT-PCR system (Life Technologies). For quantitative PCR (qPCR), cDNA was amplified using a SureCycler 8800 (Agilent Technologies, Santa Clara, CA, USA) and 2X TOPsimple ${ }^{\mathrm{TM}}$ DyeMIX-nTaq (Enzynomics, Seoul, Korea), according to the manufacturer's instructions. Gene expression was determined using agarose gel electrophoresis. GAPDH was used as the internal controls in the reactions for normalization. Primer used for $\mathrm{qPCR}$ 
were as follows: for caspase-3, forward primer, 5'-TCTTGG CGAAATTCAAAGGATGGC-3' and reverse primer, 5'-TT TGTGAGCATGGAAACAATACATGG-3'; for caspase-3, forward primer, 5'-GGCTTTGACCACGACCTTTGAAGA-3' and reverse primer, 5'-GGAAGGGCACTTCAAACCAGTG AA-3'; for FasL, forward primer, 5'-GCTGGAGTCATGACA CTAAGTCAA-3' and reverse primer, 5'-CTCTGCAAGAGT ACAAAGATTGGC-3'; for GAPDH, forward primer, 5'-AGCC TCAAGATCATCAGCAATG-3' and reverse primer, 5'-ATG GACTGTGGTCATGAGTCCTT-3'.

Immunoblotting. Cell and tissue lysates were prepared using modified radioimmunoprecipitation assay buffer (1 M Tris-HCl, $150 \mathrm{mM} \mathrm{NaCl}, 1 \%$ Triton X-100, 2 mM EDTA) with protease inhibitor and phosphatase inhibitor cocktail (both from Sigma, USA). Total protein concentrations of the cell lysates were determined by bicinchoninic acid protein assays (Pierce). Equal amounts of protein were resolved by $10 \%$ sodium dodecyl sulfate-polyacrylamide gel electrophoresis and transferred to nitrocellulose membranes for immunoblotting analyses. After blocking with $5 \%$ bovine serum albumin (BSA) in TBS-T at room temperature for $1 \mathrm{~h}$, the membranes were sequentially blotted with primary antibodies at $4^{\circ} \mathrm{C}$ overnight. After rinsing in TBS-T, the membranes were incubated with HRP-conjugated secondary antibody at room temperature for $1 \mathrm{~h}$. Immunoreactivity was visualized using the ECL system (Amersham Biosciences).

Statistical analysis. Data are expressed as the means \pm SD of 3 individual experiments performed in triplicate. Statistical analysis was performed using the Student's t-test, and $\mathrm{p}<0.05$ was considered to indicate a statistically significant result.

\section{Results}

Lico-A suppresses the viability of KB oral cancer cells, but does not affect the viability of NHOKs. The effect of Lico-A on the viability of KB oral cancer cells and NHKOs was assessed by the MTT assay. As shown in Fig. 1A, Lico-A did not have an effect on the NHKOs, which are primary normal oral keratinocytes originating from the human oral cavity, when compared to the control. In contrast, KB oral cancer cells stimulated with different concentrations of Lico-A for $24 \mathrm{~h}$ exhibited-significantly decreased cell viability when compared to the control (Fig. 1B). Particularly, 50 and $100 \mu \mathrm{M}$ Lico-A decreased the cell viability by $\sim 50$ and $80 \%$ when compared with the control. These data indicate that the $\mathrm{IC}_{50}$ value of Lico-A in $\mathrm{KB}$ oral cancer cells was $\sim 50 \mu \mathrm{M}$.

Lico-A induces apoptotic phenomena, such as DNA fragmentation, chromatin condensation and activation of caspase-3, in $\mathrm{KB}$ oral cancer cells. Following stimulation of $\mathrm{KB}$ oral cancer cells with Lico-A for $24 \mathrm{~h}$, morphological changes, such as shrinkage, aggregation and the increase in detachment of cells from the surface of culture vessels, were observed using microscopy. As shown in Fig. 1D, genomic DNA isolated from $\mathrm{KB}$ oral cancer cells stimulated with Lico-A clearly showed the formation of DNA trailing when compared to the control. These data indicate that DNA breakage or fragmentation, which only occurs when cell apoptosis takes place, was induced by
Lico-A. To observe the morphological nuclear change in KB oral cancer cells stimulated with Lico-A, we performed nuclear staining using DAPI. As shown in Fig. 1E, the population of $\mathrm{KB}$ oral cancer cells that attached to the surface of the culture vessel was significantly decreased after Lico-A stimulation. Furthermore, the number of condensed and fragmented nuclei was significantly increased in KB oral cancer cells following stimulation with Lico-A (Fig. 1E). Furthermore, to confirm the Lico-A-induced apoptosis, caspase-3 intracellular activity assay was performed using capsase-3/-7 PhiPhiLux staining. As shown in Fig. 1F, activated caspase-3 was significantly detected in the cytosol of KB oral cancer cells stimulated with Lico-A for $24 \mathrm{~h}$ in a dose-dependent manner. In contrast, in $\mathrm{KB}$ oral cancer cells stimulated without Lico-A as the control activated caspase- 3 was not observed. These data suggest that Lico-A-induced cell death was mediated by apoptosis.

Lico-A increases the number of apoptotic cells in a time-dependent manner. To determine whether Lico-A-induced cell death is associated with the induction of apoptosis, $\mathrm{KB}$ oral cancer cells were stimulated with $50 \mu \mathrm{M}$ Lico-A for 12 and $24 \mathrm{~h}$ and subsequently co-stained with the apoptotic markers Annexin V-FITC and necrotic marker PI. As shown in Fig. 2, the number of apoptotic cells at the early stage of apoptosis was gradually increased by 14.08 and $28.19 \%$ at 12 and $24 \mathrm{~h}$, respectively, in a time-dependent manner. In addition, as shown in Fig. 2, the percentage of Annexin V-FITC-positive cells at both the early and late stage of apoptosis was gradually increased up to $29.95 \%$ at $24 \mathrm{~h}$ when compared with the control.

Lico-A-induced apoptosis is mediated via the FasL/PARP axis. To verify the mechanism by which Lico-A induces the apoptosis of KB oral cancer cells, qPCR and immunoblotting were performed to measure the expression of apoptosis-related genes at both the mRNA and protein levels. As shown in Fig. 3A, FasL, which is an apoptotic ligand and triggers the extrinsic apoptotic pathway, was significantly induced by Lico-A in KB oral cancer cells. Subsequently, caspase-8 and -3, which are downstream targets of FasL, were significantly induced by Lico-A at the mRNA level. However, apoptosis requires the activation of the caspase cascade and PARP. As shown in Fig. 3B, FasL was significantly expressed in KB cells following Lico-A treatment in a dose-dependent manner. Sequentially, upregulated FasL by LicoA triggered the cleavage of pro-caspase- 8 into cleaved (activated) caspase- 8 (43 kDa). In the next step, cleaved caspase- 8 induced the cleavage of pro-caspase- 3 as its downstream target. Activated caspase- 3 (17 and $19 \mathrm{kDa}$ ) gradually increased in a dose-dependent manner. Furthermore, cleaved PARP (85 and $25 \mathrm{kDa}$ ) was increased by activated caspase- 3 when compared with the control. $\beta$-actin was used as an internal control for normalization. Therefore, these data clearly indicate that Lico-A-induced apoptosis in KB oral cancer cell was mediated by the extrinsic apoptotic pathway via the FasL/PARP axis.

Lico-A-induced apoptosis is regulated by the activation of caspases in KB oral cancer cells. Apoptotic signals are mediated by the activation of the caspase cascade, which is a key hallmark of apoptosis. We, therefore, determined a 

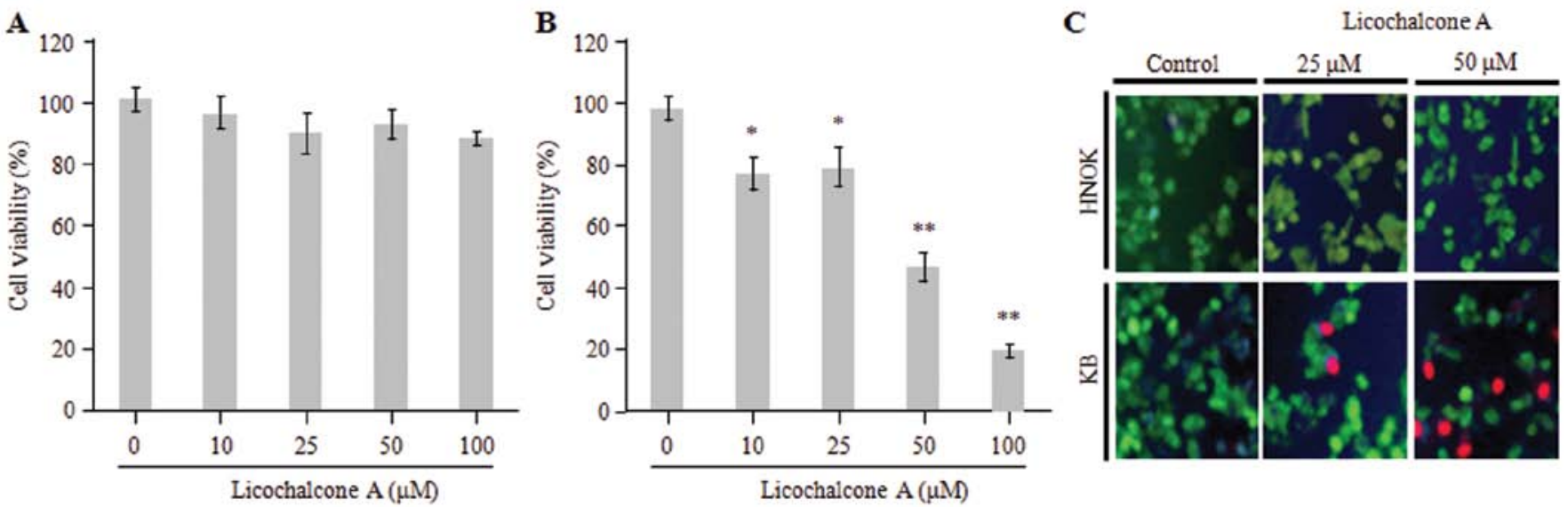

D

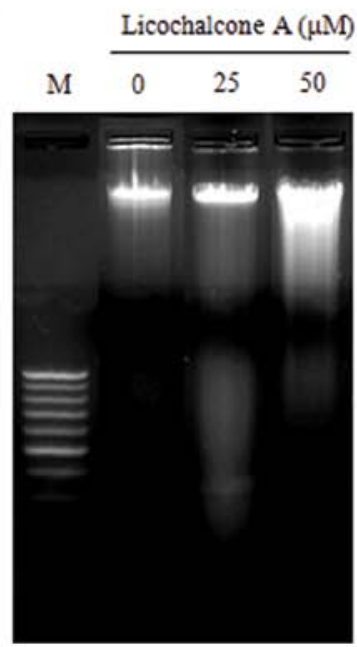

$\mathbf{E}$
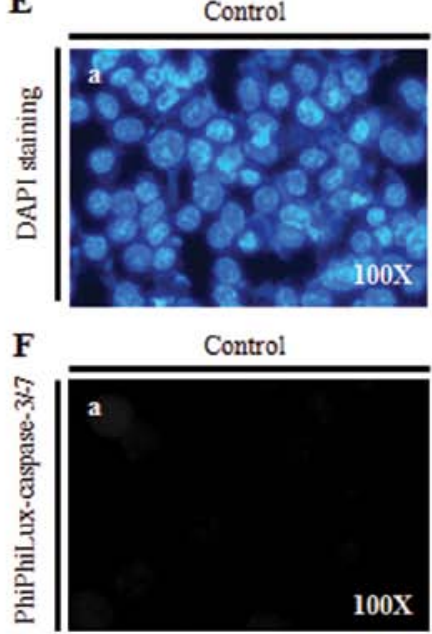

$25 \mu \mathrm{M}$ Licochalcone A

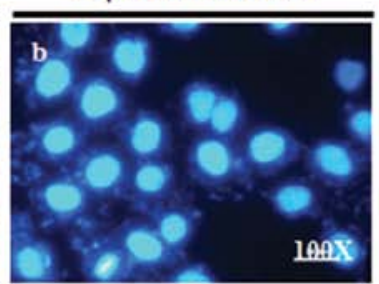

$25 \mu \mathrm{MLicochalcone} \mathrm{A}$

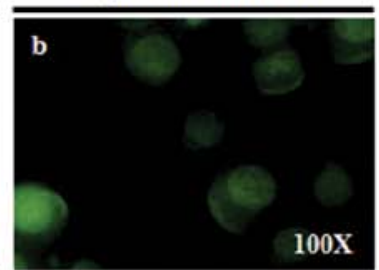

$50 \mu \mathrm{M}$ Licochalcone A

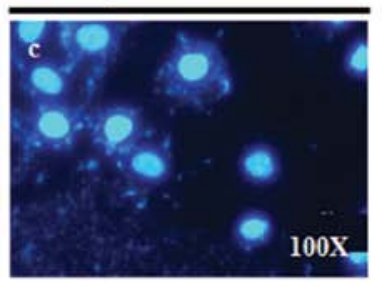

$50 \mu \mathrm{MLicochalcone} \mathrm{A}$

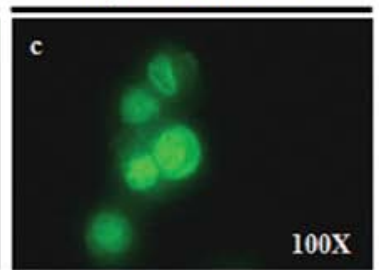

Figure 1. Licochalcone A selectively induces the apoptosis of human KB oral cancer calls, but not human normal oral keratinocytes. (A) Human normal oral keratinocytes and (B) KB oral cancer cells were stimulated with different doses of licochalcone A (0, 20, 25, 50 and $100 \mu \mathrm{M})$ for $24 \mathrm{~h}$. After the indicated stimulating condition, cell viability was measured using an MTT assay, and cell viability was quantified by measuring the optical density at 570 nm. The data represent triplicate results of 3 independent experiments and are expressed as means $\pm \mathrm{SD}\left({ }^{*} \mathrm{p}<0.05\right.$ and ${ }^{* *} \mathrm{p}<0.01$ as compared to the control). (C) Cell survival assay using green calcein AM for live cells (green color) and ethidium bromide homodimer 1 for dead cell (red color). (D) For analysis of genomic DNA fragmentation, KB cells were stimulated with different doses of licochalcone A for $24 \mathrm{~h}$, harvested, and the genomic DNA was isolated. Genomic DNA isolated from the KB cells was electrophoresed on 1.5\% agarose gel containing ethidium bromide and visualized using UV. (E) DAPI staining to identify nuclear condensation. KB cells were stimulated with different doses of licochalcone A for $24 \mathrm{~h}$, fixed with $4 \%$ paraformaldehyde and nuclear staining was performed using DAPI. The cells stained by DAPI were observed under a fluorescence microscope. (F) Caspase-3 intracellular activity assay. KB cells were stimulated with different doses of licochalcone A for $24 \mathrm{~h}$, fixed with $4 \%$ paraformaldehyde and nuclear staining was performed using PhiPhiLux-caspase-3/-7. The cells stained with DAPI were observed under a fluorescence microscope. DAPI, 4',6-diamidino-2-phenylindole.

direct involvement of caspase activation in Lico-A-induced $\mathrm{KB}$ oral cancer cell apoptosis. As shown in Fig. 4A, $50 \mu \mathrm{M}$ of Z-VAD-fmk, a pan-caspase inhibitor, had no effect on the cell viability in $\mathrm{KB}$ oral cancer cells similar to the non-treated control. In addition, $50 \mu \mathrm{M}$ Lico-A consistently decreased the cell viability by $\sim 50 \%$ (Fig. 1B). However, Lico-A-induced cell cytotoxicity in $\mathrm{KB}$ oral cancer cells was partially recovered by treatment with Z-VAD-fmk as compared with Lico-A stimulation only. Furthermore, Z-VAD-fmk treatment led to inhibition of Lico-A-induced caspase-3 and PARP activation (Fig. 4B). These data indicate that the Lico-A-induced apoptosis was regulated by the activation of the caspase cascade in $\mathrm{KB}$ oral cancer cells.

ERK $K^{M A P K}$ and $p 38^{M A P K}$ pathways are required for Lico-A-induced FasL upregulation and subsequent apoptosis in $K B$ oral cancer cells. To elucidate the apoptotic signaling pathways involved in the Lico-A-induced FasL expression in $\mathrm{KB}$ oral cancer cells, we examined the phosphorylation of MAPK signaling pathways that have previously been linked to FasL expression in the apoptosis of cancer cells. As shown in Fig. 5A, $50 \mu \mathrm{M}$ Lico-A activated both ERK and p38, as reflected by phosphorylation within $5 \mathrm{~min}$ and sustained activation for $30 \mathrm{~min}$ after stimulation with Lico-A. There was no significant activation of JNK ${ }^{\text {MAPK }}$ by Lico-A in KB oral cancer cells. To determine which pathway was involved in Lico-Ainduced FasL expression, we performed immunoblotting in the presence or absence of pathway-specific inhibitors of either ERK (PD98059) or p38 (SB203580). The presence of each inhibitor suppressed the Lico-A-mediated FasL expression in $\mathrm{KB}$ oral cancer cells (Fig. 5B). Collectively, these findings demonstrated that Lico-A-induced apoptosis of KB oral cancer cells was mediated by the upregulation of FasL via the activation of both the ERK ${ }^{\mathrm{MAPK}}$ and $\mathrm{p} 38^{\mathrm{MAPK}}$ signaling pathways. 

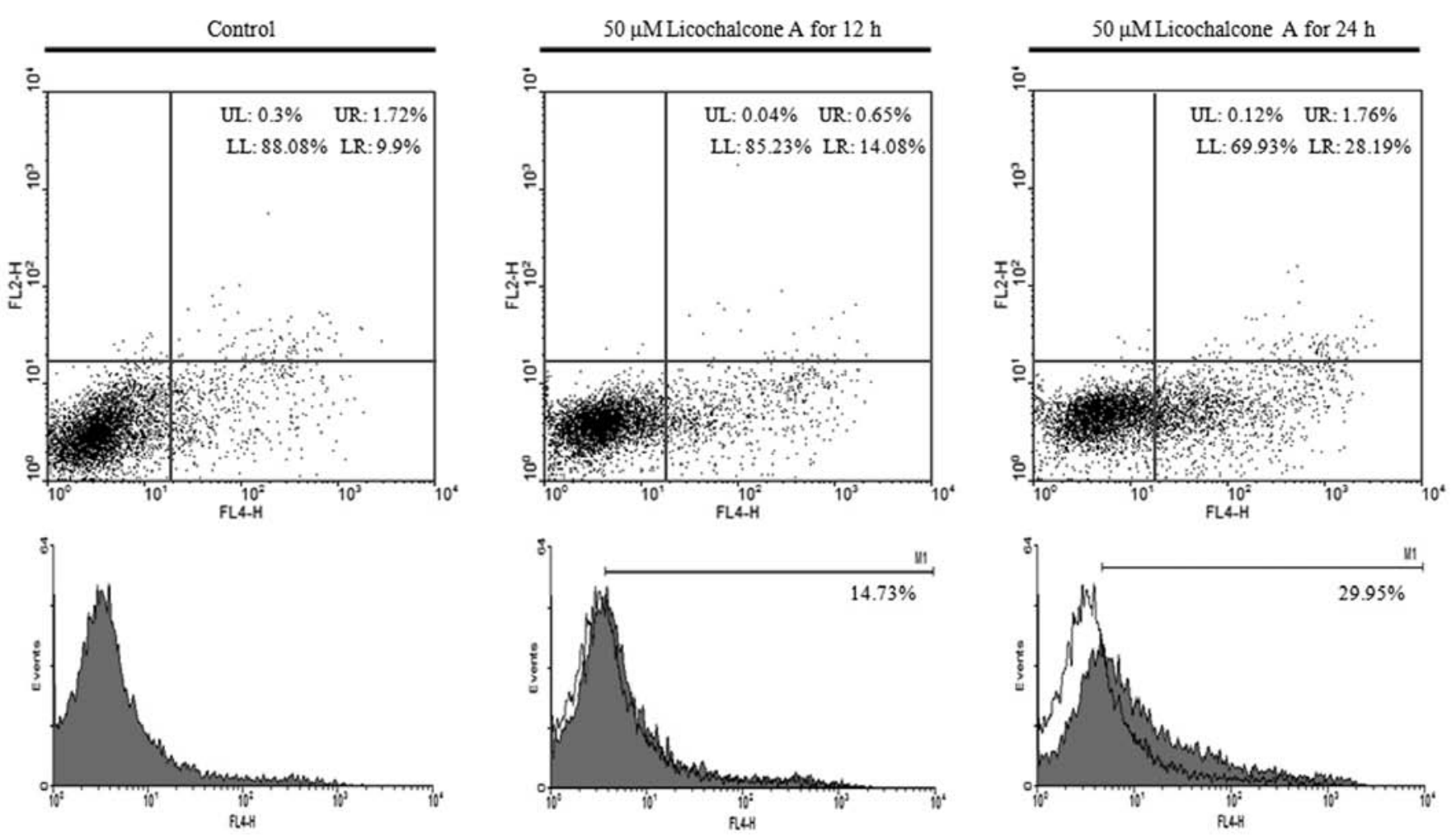

Figure 2. Licochalcone A treatment increases the apoptotic population in KB oral cancer cells. To assess the licochalcone A-induced KB oral cancer cell apoptosis, FACS analysis was performed by Annexin V and PI staining. KB oral cancer cells were cultured in complete medium for $24 \mathrm{~h}$ and stimulated with $50 \mu \mathrm{M}$ licochalcone $\mathrm{A}\left(\mathrm{IC}_{50}\right.$ concentration) for 12 and $24 \mathrm{~h}$. After stimulation, the cells were analyzed by flow cytometry.

$\mathbf{A}$

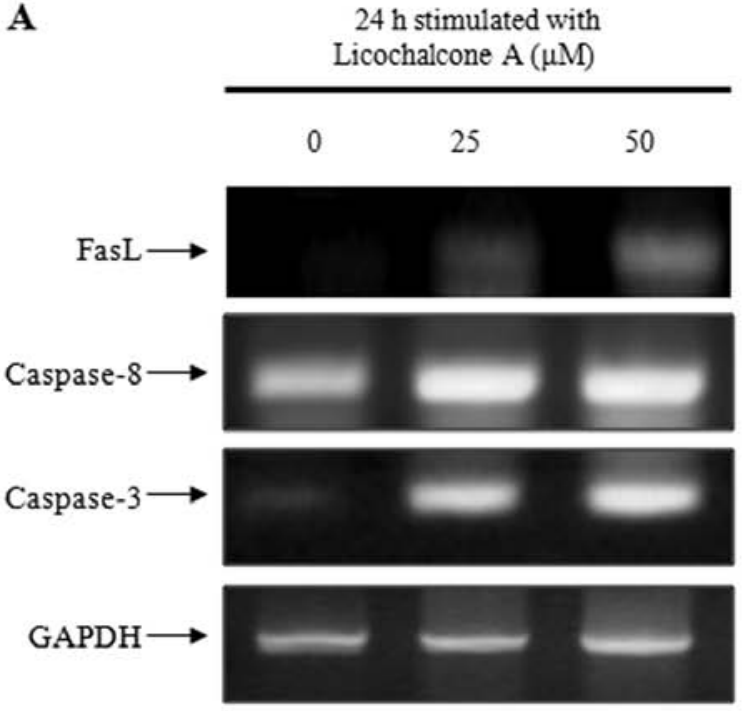

B
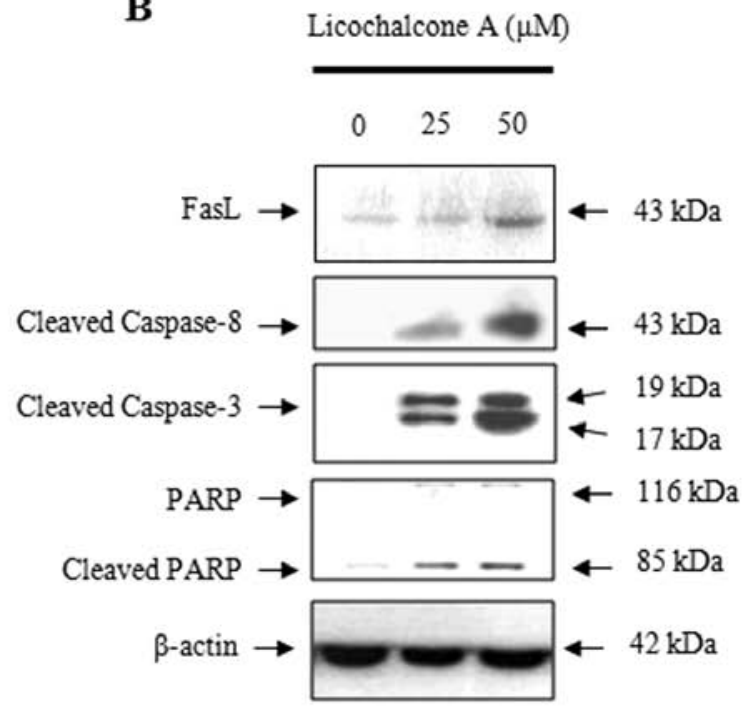

Figure 3. Licochalcone A-induced apoptosis in KB oral cancer cells is mediated by expression and activation of caspases. (A) Total RNA from KB oral cancer cells stimulated with licochalcone A for $24 \mathrm{~h}$ was isolated using TRIzol reagent. cDNA synthesis was carried out with $1 \mu \mathrm{g}$ total RNA using reverse transcriptase. qPCR was performed using synthesized cDNA. The amplified PCR product was then electrophoresed on 1\% agarose gel. (B) Licochalcone A activated the extrinsic apoptosis signaling pathway in KB oral cancer cells. KB oral cancer cells were stimulated with 25 and $50 \mu \mathrm{M}$ licochalcone A for $24 \mathrm{~h}$, harvested and lysed using cell lysate buffer. Protein quantification and western blotting were performed. qPCR, quantitative PCR.

\section{Discussion}

Oral cancers are common malignancies which have emerged as major international health issues. Global cancer statistics has revealed that the annual incidence of oral cancer exceeds
270,000 cases worldwide (27). Although the clinical treatment for oral cancer has improved, the 5-year survival rate for patients with oral cancer is approximately $50 \%$ (28). Even though chemotherapy is one of the important therapeutic strategies for oral cancer, it is still limited by various side-effects 
A

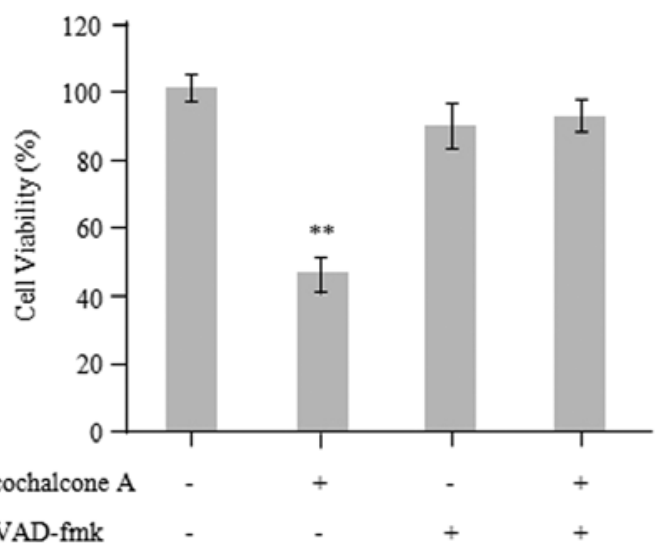

$50 \mu \mathrm{M}$ Licochalcone A

$50 \mu \mathrm{MZ}$-VAD-fmk

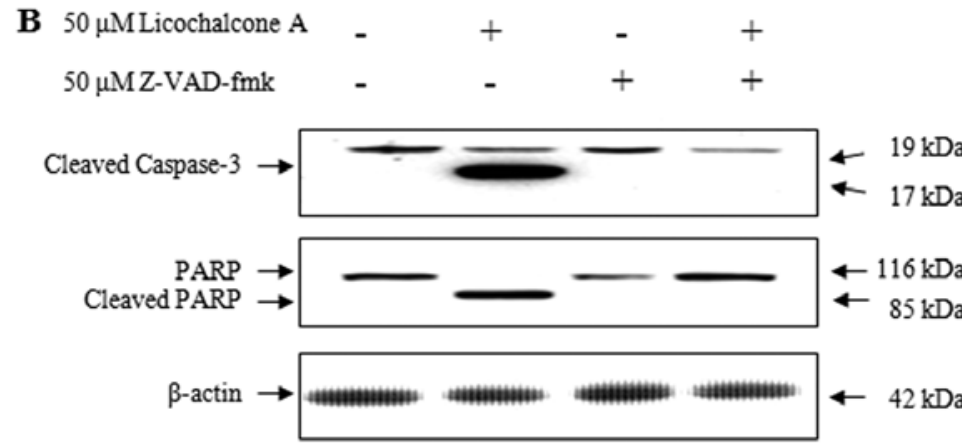

Figure 4. Licochalcone A-induced apoptosis in KB oral cancer cells is mediated by caspase activation. (A) Z-VAD-fmk, a caspase inhibitor, inhibited licochalcone A-induced KB cell apoptosis. KB cells were stimulated with $50 \mu \mathrm{M}$ licochalcone A present with/without $50 \mu \mathrm{M} \mathrm{Z-VAD-fmk} \mathrm{for} 24 \mathrm{~h}$. After stimulation, cell viability was assessed by MTT assay. (B) The increased expression and activation of apoptosis-related proteins by licochalcone A in KB oral cancer cells were suppressed by Z-VAD-fmk. KB cells were stimulated with $50 \mu \mathrm{M}$ licochalcone A present with/without $50 \mu \mathrm{M} \mathrm{Z-VAD-fmk}$ for $24 \mathrm{~h}$, harvested, and lysed using cell lysate buffer. Protein quantification and immunoblotting were performed.

A

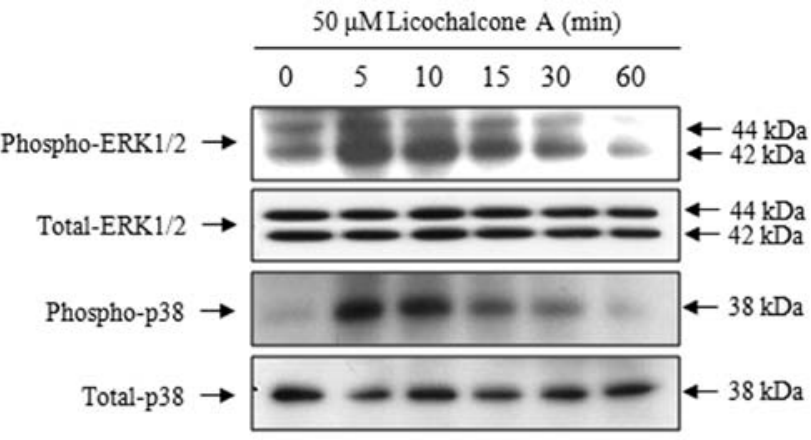

B

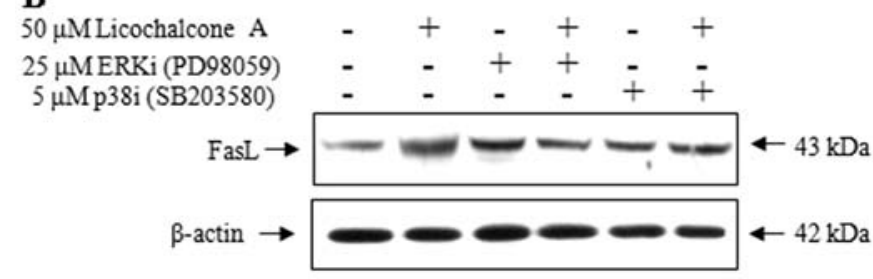

Figure 5. Licochalcone A-induced apoptosis in KB oral cancer cells is associated with activation of the MAPK signaling pathway. (A) Serum-starved KB oral cancer cells were stimulated with $50 \mu \mathrm{M}$ licochalcone A for the indicated periods of time. Cell lysates were then prepared and analyzed by immunoblotting with specific anti-phospho-ERK, anti-total-ERK, anti-phospho-p38 and anti-total p38 antibodies. (B) Serum starved KB oral cancer cells were treated with $50 \mu \mathrm{M}$ licochalcone $\mathrm{A}$ in the presence or absence of the ERK $^{\mathrm{MAPK}}$ pathway-specific inhibitor PD98059 (ERKi; $25 \mu \mathrm{M}$ ) and the p38 ${ }^{\mathrm{MAPK}}$ pathway-specific inhibitor SB203580 (p38i; $5 \mu \mathrm{M}$ ). The cells were harvested $24 \mathrm{~h}$ after the initiation of each treatment to perform immunoblotting for Fas. $\beta$-actin was used as a control for normalization.

such as high toxicity and drug tolerance. Therefore, there is an urgent demand for the development of effective clinical drugs with fewer side-effects. Based on these requirements, natural therapies, which use the natural compounds derived from medicinal plants and traditional Oriental medicine, are being developed to overcome the side-effects of chemotherapeutic reagents.
Glycyrrhiza uralensis Fischer is one of the representative medicinal herbal plants for the treatment of sore throat, cough, bronchitis, peptic ulcers, arthritis and allergic disease in traditional Oriental medicine $(29,30)$. In addition, Lico-A, the major bioactive compound isolated from Glycyrrhiza sp., has been reported to have various biological activities such as anti-inflammatory $(31,32)$, anti-microbial (33), anti-angiogenic (34), anti-obesity (35) and osteogenic effects (4). In the present study, we demonstrated that Lico-A suppressed the proliferation and induced the apoptosis of KB oral cancer cells via death receptor-mediated caspase activation.

First, we assessed the cell cytotoxicity of Lico-A in both human KB oral cancer cells and primary human oral normal keratinocytes to determine the possibility of its use as a potential chemotherapeutic agent for treating oral cancer. As shown in Fig. 1, the various concentrations of Lico-A did not affect the cell viability in primary human normal oral keratinocytes. In contrast, cell cytotoxicity was significantly increased in human KB oral cancer cells stimulated with Lico-A in a dose-dependent manner. Notably, the cell viability of KB cells was effectively decreased by $\sim 50 \%$ at the concentration of $50 \mu \mathrm{M}$ Lico-A for $24 \mathrm{~h}$. Xiao et al also reported that the cell cytotoxicity of gastric cancer cells including MKN-28, AGS and MKN-45 was significantly increased following treatment with $\sim 50 \mu \mathrm{M}$ of Lico-A for $24 \mathrm{~h}$ (8). In contrast, Lico-A exhibited less cytotoxicity to normal human gastric mucosal cells similar to our demonstrated results. These data clearly suggest that Lico-A enhanced the cancer cellspecific cytotoxicity without affecting normal cells. Next, to investigate the mechanism by which Lico-A induces human KB oral cancer cell death, we analyzed DNA fragmentation, morphological alteration and caspase-3 expression following Lico-A stimulation. As shown in Fig. 1D, Lico-A significantly induced DNA fragmentation in human KB oral cancer cells dose-dependently. Furthermore, the morphological alterations including chromatin condensation and a decrease in the cell population were observed in human KB oral cancer cells stained with DAPI after Lico-A treatment (Fig. 1E). As a key feature of apoptotic cell death, both DNA fragmentation and 
chromatin condensation were observed in human KB oral cancer cells stimulated with Lico-A. During apoptotic events, DNA fragmentation is mediated by caspase-activated DNase, which is an endonuclease found in the extrinsic apoptotic pathway and is activated by caspase-3 (36). Therefore, we performed the caspase- 3 intracellular activity assay using PhiPhiLux-caspase-3 to detect the activated caspase- 3 in human KB oral cancer cells stimulated with Lico-A. As shown in Fig. 1F, activated caspase-3 was significantly detected intracellularly in human KB oral cancer cells stimulated with Lico-A. In addition, the apoptotic population at both the early and late stages of apoptosis was significantly increased in human $\mathrm{KB}$ oral cancer cells stimulated with $50 \mu \mathrm{M}$ of Lico-A in a time-dependent manner (Fig. 2). These data indicate that Lico-A significantly induced the activation of caspase- 3 . Furthermore, activated caspase- 3 induced the cleavage of the inhibitor of caspase-activated DNase for formatting the caspase-activated DNase. Subsequently, caspase-activated DNase fragmented the genomic DNA of human KB oral cancer cells stimulated with Lico-A. However, these data are consistent indicating that Lico-A-induced human KB oral cancer cell death is closely associated with apoptosis via activation of caspases.

The factor associated suicide ligand (FasL), an important regulatory factor of apoptosis, initiates the death receptormediated extrinsic apoptotic pathway through the activation of caspase- 8 and -3 and PARP, sequentially, after binding with receptor FasR spanned on the surface of target cells $(37,38)$. As shown in Fig. 3, the expression of FasL was significantly upregulated by Lico-A in the KB oral cancer cells. Subsequently, upregulated FasL triggered a caspase cascade and subsequently resulted in the activation of apoptotic factors, including caspase- 8 and -3 . Finally, activated caspase- 3 cleaved its major substrate PARP resulting in consequent apoptosis. These data clearly suggest that caspase- 3 is activated in response to Lico-A in KB oral cancer cells. Therefore, to further evaluate the role of caspase-3 in Lico-A-induced apoptosis, KB oral cancer cells were stimulated with Z-VAD-fmk, a specific caspase-3 inhibitor, to suppress the cleavage of caspase-3. As shown in Fig. 4A, Z-VAD-fmk significantly inhibited the LicoA-induced apoptosis of KB oral cancer cells. Furthermore, the activation of caspase-3 and its major substrate PARP in KB oral cancer cells stimulated with Lico-A was significantly suppressed by Z-VAD-fmk, indicating that Lico-A-induced apoptosis was dependent on caspase-3 activity (Fig. 4B).

To further investigate the signaling pathway involved in Lico-A-induced apoptosis in KB oral cancer cells, we assessed the effect of Lico-A on mitogen-activating protein kinases, which are associated with the apoptotic signaling pathway. As shown in Fig. 5A, Lico-A induced the phosphorylation of ERK and p38 in a time-dependent manner. However, we did not detect any significant phosphorylation of JNK following Lico-A treatment in KB oral cancer cells. Therefore, to confirm the role of ERK and p38 inhibition in Lico-A-induced FasL expression, KB oral cancer cells were pre-stimulated with pharmacological inhibitors (PD98059 for ERK and SB203580 for p38) for $2 \mathrm{~h}$ to turn off each MAPK signaling and then Lico-A was administered for $24 \mathrm{~h}$. Lico-A-induced FasL expression was significantly suppressed by the inhibition of ERK and p38 signaling (Fig. 5B). These results indicate that ERK and p38 are required for the Lico-A-induced FasL expression and apoptosis in KB oral cancer cells. Binding of FasL to its receptor FasR has been shown to activate MAPK, and its activation is required for apoptosis of human hepatocellular carcinoma Huh7 cells (39). Therefore, we demonstrated that Lico-A induced the extrinsic apoptotic signaling pathway in KB oral cancer cells via the upregulation of FasL through both ERK and p38 activation. In conclusion, Lico-A may be developed as a chemotherapeutic agent for the management of oral cancer.

\section{References}

1. Feldman $\mathrm{M}$ and Grenier D: Cranberry proanthocyanidins act in synergy with licochalcone A to reduce Porphyromonas gingivalis growth and virulence properties, and to suppress cytokine secretion by macrophages. J Appl Microbiol 113: 438-447, 2012.

2. Messier C and Grenier D: Effect of licorice compounds licochalcone A, glabridin and glycyrrhizic acid on growth and virulence properties of Candida albicans. Mycoses 54: e801-e806, 2011.

3. Tadigoppula N, Korthikunta V, Gupta S, et al: Synthesis and insight into the structure-activity relationships of chalcones as antimalarial agents. J Med Chem 56: 31-45, 2013.

4. Kim SN, Bae SJ, Kwak HB, et al: In vitro and in vivo osteogenic activity of licochalcone A. Amino Acids 42: 1455-1465, 2012

5. Kim YH, Shin EK, Kim DH, Lee HH, Park JH and Kim JK: Antiangiogenic effect of licochalcone A. Biochem Pharmacol 80: 1152-1159, 2010.

6. Szliszka E, Jaworska D, Ksek M, Czuba ZP and Król W: Targeting death receptor TRAIL-R2 by chalcones for TRAIL-induced apoptosis in cancer cells. Int J Mol Sci 13: 15343-15359, 2012.

7. Lee CS, Kwak SW, Kim YJ, et al: Guanylate cyclase activator YC-1 potentiates apoptotic effect of licochalcone A on human epithelial ovarian carcinoma cells via activation of death receptor and mitochondrial pathways. Eur J Pharmacol 683: 54-62, 2012.

8. Xiao XY, Hao M, Yang XY, et al: Licochalcone A inhibits growth of gastric cancer cells by arresting cell cycle progression and inducing apoptosis. Cancer Lett 302: 69-75, 2011.

9. Kim JK, Shin EK, Park JH, Kim YH and Park JH: Antitumor and antimetastatic effects of licochalcone A in mouse models. J Mol Med 88: 829-838, 2010.

10. Messier C, Epifano F, Genovese S and Grenier D: Licorice and its potential beneficial effects in common oro-dental diseases. Oral Dis 18: 32-39, 2012.

11. Napier SS and Speight PM: Natural history of potentially malignant oral lesions and conditions: an overview of the literature. J Oral Pathol Med 37: 1-10, 2008.

12. Moreno-López LA, Esparza-Gómez GC, González-Navarro A Cerero-Lapiedra R, González-Hernández MJ and Domínguez-Rojas V: Risk of oral cancer associated with tobacco smoking, alcohol consumption and oral hygiene: a case-control study in Madrid, Spain. Oral Oncol 36: 170-174, 2000.

13. Lin WJ, Jiang RS, Wu SH, Chen FJ and Liu SA: Smoking, alcohol, and betel quid and oral cancer: a prospective cohort study. J Oncol 2011: 525976, 2011.

14. Humphris GM, Freeman R and Clarke HM: Risk perception of oral cancer in smokers attending primary care: a randomised controlled trial. Oral Oncol 40: 916-924, 2004.

15. Shiu MN and Chen TH: Impact of betel quid, tobacco and alcohol on three-stage disease natural history of oral leukoplakia and cancer: implication for prevention of oral cancer. Eur J Cancer Prev 13: 39-45, 2004.

16. Shah FD, Begum R, Vajaria BN, et al: A review on salivary genomics and proteomics biomarkers in oral cancer. Indian J Clin Biochem 26: 326-334, 2011.

17. Yeh CC, Tseng CN, Yang JI, et al: Antiproliferation and induction of apoptosis in Ca9-22 oral cancer cells by ethanolic extract of Gracilaria tenuistipitata. Molecules 17: 10916-10927, 2012.

18. Yeh CC, Yang JI, Lee JC, et al: Anti-proliferative effect of methanolic extract of Gracilaria tenuistipitata on oral cancer cells involves apoptosis, DNA damage, and oxidative stress. BMC Complement Altern Med 12: 142, 2012.

19. Kang JW, Kim JH, Song K, Kim SH, Yoon JH and Kim KS: Kaempferol and quercetin, components of Ginkgo biloba extract (EGb 761), induce caspase-3-dependent apoptosis in oral cavity cancer cells. Phytother Res 24 (Suppl 1): S77-S82, 2010. 
20. Choi ES, Cho SD, Jeon JG and Cho NP: The apoptotic effect of the hexane extract of Rheum undulatum L. in oral cancer cells through the down-regulation of specificity protein 1 and survivin. Lab Anim Res 27: 19-24, 2011.

21. Chen YJ, Liao CT, Chen PJ, et al: Downregulation of Ches 1 and other novel genes in oral cancer cells chronically exposed to areca nut extract. Head Neck 33: 257-266, 2011.

22. Derradji H and Baatout S: Apoptosis: a mechanism of cell suicide. In Vivo 17: 185-192, 2003.

23. Lin JW, Chen JT, Hong CY, et al: Honokiol traverses the bloodbrain barrier and induces apoptosis of neuroblastoma cells via an intrinsic bax-mitochondrion-cytochrome c-caspase protease pathway. Neuro Oncol 14: 302-314, 2012.

24. Oh HL, Lim H, Park Y, et al: HY253, a novel compound isolated from Aralia continentalis, induces apoptosis via cytochrome $c$-mediated intrinsic pathway in HeLa cells. Bioorg Med Chem Lett 19: 797-799, 2009.

25. Nyåkern M, Cappellini A, Mantovani I and Martelli AM: Synergistic induction of apoptosis in human leukemia T cells by the Akt inhibitor perifosine and etoposide through activation of intrinsic and Fas-mediated extrinsic cell death pathways. Mol Cancer Ther 5: 1559-1570, 2006.

26. Kim JS,Ellman MB, An HS, van Wijnen AJ, Borgia JA and Im HJ: Insulin-like growth factor 1 synergizes with bone morphogenetic protein 7-mediated anabolism in bovine intervertebral disc cells Arthritis Rheum 62: 3706-3715, 2010.

27. Partridge M, Li SR, Pateromichelakis S, et al: Detection of minimal residual cancer to investigate why oral tumors recur despite seemingly adequate treatment. Clin Cancer Res 6 : 2718-2725, 2000.

28. Parkin DM, Bray F, Ferlay J and Pisani P: Global cancer statistics, 2002. CA Cancer J Clin 55: 74-108, 2005.

29. Chung WT, Lee SH, Kim JD, et al: Effect of the extracts from Glycyrrhiza uralensis Fisch on the growth characteristics of human cell lines: anti-tumor and immune activation activities. Cytotechnology 37: 55-64, 2001.
30. Tang J, Song X, Zhu M and Zhang J: Study on the pharmacokinetics drug-drug interaction potential of Glycyrrhiza uralensis, a traditional Chinese medicine, with lidocaine in rats. Phytother Res 23: 603-607, 2009.

31. Franceschelli S, Pesce M, Vinciguerra I, et al: Licocalchone-C extracted from Glycyrrhiza glabra inhibits lipopolysaccharideinterferon- $\gamma$ inflammation by improving antioxidant conditions and regulating inducible nitric oxide synthase expression. Molecules 16: 5720-5734, 2011.

32. Wu TY, Khor TO, Saw CL, et al: Anti-inflammatory/Anti-oxidative stress activities and differential regulation of Nrf2-mediated genes by non-polar fractions of tea Chrysanthemum zawadskii and licorice Glycyrrhiza uralensis. AAPS J 13: 1-13, 2011.

33. Lee JW, Ji YJ, Yu MH, et al: Antimicrobial effect and resistant regulation of Glycyrrhiza uralensis on methicillin-resistant Staphylococcus aureus. Nat Prod Res 23: 101-111, 2009.

34. Kim JM, Kim KS, Lee YW, et al: Anti-angiogenic effects of water extract of a formula consisting of Pulsatilla koreana, Panax ginseng and Glycyrrhiza uralensis. Zhong Xi Yi Jie He Xue Bao 9: 1005-1013, 2011.

35. Birari RB, Gupta S, Mohan CG and Bhutani KK: Antiobesity and lipid lowering effects of Glycyrrhiza chalcones: experimental and computational studies. Phytomedicine 18: 795-801, 2011.

36. Walker PR, Weaver VM, Lach B, LeBlanc J and Sikorska M Endonuclease activities associated with high molecular weight and internucleosomal DNA fragmentation in apoptosis. Exp Cell Res 213: 100-106, 1994.

37. Huang DC, Hahne M, Schroeter M, et al: Activation of Fas by FasL induces apoptosis by a mechanism that cannot be blocked by Bcl-2 or Bcl- $x_{L}$. Proc Natl Acad Sci USA 96: 14871-14876, 1999.

38. Herrnring C, Reimer T, Jeschke U, et al: Expression of the apoptosis-inducing ligands FasL and TRAIL in malignant and benign human breast tumors. Histochem Cell Biol 113: 189-194, 2000.

39. Wang WZ, Li L, Liu MY, et al: Curcumin induces FasL-related apoptosis through p38 activation in human hepatocellular carcinoma Huh7 cells. Life Sci 92: 352-358, 2013. 